\title{
Parametric Estimation of Wave Dispersion for System Identification of Building Structures
}

\author{
Hamed Ebrahimian ${ }^{1}$, Monica Kohler ${ }^{2}$, Anthony Massari ${ }^{3}$, Domniki Asimaki ${ }^{4}$ \\ ${ }^{1}$ Postdoctoral Scholar, Department of Mechanical and Civil Engineering, Caltech, Pasadena, CA, USA. \\ ${ }^{2}$ Research Assistant Professor, Department of Mechanical and Civil Engineering, Caltech, Pasadena, CA, USA. \\ ${ }^{3}$ Ph.D. Candidate, Department of Mechanical and Civil Engineering, Caltech, Pasadena, CA, USA. \\ ${ }^{4}$ Professor, Department of Mechanical and Civil Engineering, Caltech, Pasadena, CA, USA.
}

\begin{abstract}
The linear-elastic response of a building structure subjected to an earthquake base excitation can be approximated as the response of a continuous, spatially inhomogenous, dispersive, viscoelastic solid subjected to vertically incident plane shear waves. The frequency-dependent phase velocity and attenuation of seismic energy at different wavelengths, together with the inertial properties of the multilayer solid characterize the response of the building structure. The objective of this study is to identify the structural system by estimating the parameters that characterize the propagation of seismic waves in an equivalent multilayer viscoelastic solid. To pursue this objective, first, the measured dynamic responses of a building structure are used to derive the frequency response functions (FRFs) of the floor absolute acceleration with respect to the base excitation using a seismic interferometry approach. The FRFs obtained from the measured structural responses are then compared with the FRFs estimated using analytical models for one-dimensional shear wave propagation in a multilayer Kelvin-Voigt dispersive medium. Through a recursive Bayesian estimation approach, the parameters characterizing the phase velocity and damping ratio of the multilayer medium are estimated. This study provides a step forward in seismic interferometric identification of building structures by proposing a new method for parametric estimation of shear wave velocity and damping dispersion at the story level of a building structure. The estimated shear wave velocities before and after a damage-inducing event can be used to identify permanent loss of effective lateral stiffness of the building structure at the story level, thus can provide an alternative method for structural health monitoring and damage identification.
\end{abstract}

Keywords: Wave inversion, Dispersion, Bayesian estimation, Interferometry, Structural System Identification.

\section{INTRODUCTION}

The linear-elastic response of a building structure subjected to an earthquake base excitation can be approximated by modeling the propagation of seismic waves through a continuous, layered, viscoelastic solid. The velocity of shear waves, which are induced by seismic base excitation and propagating along the building height, can be estimated from the impulse response functions (IRFs) of the building structure, e.g., [1-6] to name only a few. Considering that the shear wave velocity is related to the lateral stiffness of the building structure, several studies have been able to detect structural damage in terms of loss of effective lateral stiffness by comparing estimated shear wave velocities from the pre- and post-damage IRFs (e.g., [79]).

In this study, an advanced sequential Bayesian estimation method for parametric estimation of wave dispersion in a continuous, multilayer, viscoelastic solid is proposed. While the proposed approach in this study is general and can be applied to other wave inversion problems, it is validated using a structural system identification case study. In this case study, a building structure is identified as a layered, dispersive, Kelvin-Voigt model, in which each layer represents a story or group of stories. Empirical functions are defined to characterize the dispersion of phase velocity and damping ratio at each layer. The parameters characterizing phase velocity and damping ratio as a function of frequency, and mass density profile along the building height are estimated and their estimation uncertainties are quantified by minimizing the discrepancies between the measured and predicted FRFs.

\section{PARAMETRIC ESTIMATION OF SEISMIC WAVE DISPERSION}

\section{One-dimensional wave propagation in a multilayered Kelvin-Voigt model}

To analyze the seismic wave propagation in a building structure, the building is modeled as a horizontally layered shear beam on a rigid base excited by vertically incident plane shear waves (Figure 1). The shear force-deformation response of each 
layer of the shear beam is characterized by a Kelvin-Voigt viscoelastic material model. The solution to the vertically propagating SH-wave in a layered shear beam for a harmonic motion of frequency $f$ can be expressed as [10]

$$
\boldsymbol{u}(\boldsymbol{z}, \boldsymbol{t})=\boldsymbol{A} \boldsymbol{e}^{i\left(2 \pi f t+k^{*} z\right)}+\boldsymbol{B} \boldsymbol{e}^{i\left(2 \pi f t-k^{*} z\right)}
$$

in which $\boldsymbol{A}, \boldsymbol{B}=$ constants representing the amplitude of the waves travelling in $-\boldsymbol{z}$ and $+\boldsymbol{z}$ (upward and downward) directions, respectively, and $\boldsymbol{k}^{*}=2 \pi \boldsymbol{f} / \boldsymbol{V}^{*}$ is the complex wave number. The term $\boldsymbol{V}^{*}=\boldsymbol{V} \sqrt{1+2 \boldsymbol{i} \xi}$ is the complex shear wave velocity and $\xi=\pi \eta \boldsymbol{f} / \boldsymbol{G}$ is the damping ratio, where $\eta$ denotes the viscosity and $\boldsymbol{G}$ denotes the shear modulus of the Kelvin-Voigt material model.

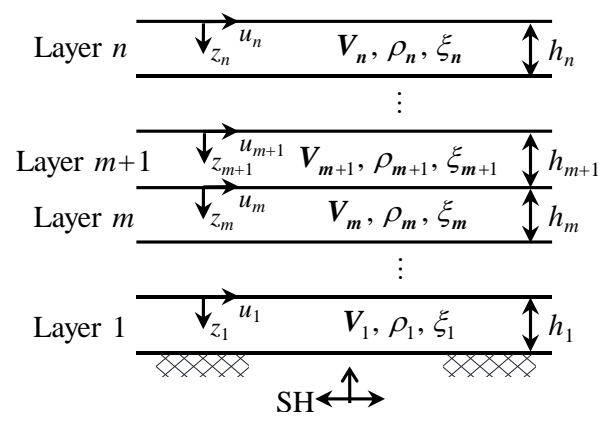

Figure 1. Multilayer Kelvin-Voigt shear beam model on a rigid base.

By introducing a local coordinate system for each layer as shown in Figure 1, the displacement and force compatibility at each layer interface yields the following two equations.

$$
\begin{aligned}
& \boldsymbol{u}_{m+1}\left(\boldsymbol{h}_{m+1}, t\right)=\boldsymbol{u}_{m}(0, \boldsymbol{t}) \Rightarrow A_{m+1} e^{i k_{m+1}^{*} h_{m+1}}+\boldsymbol{B}_{m+1} e^{-i k_{m+1}^{*} h_{m+1}}=A_{m}+\boldsymbol{B}_{m} \\
& \tau_{m+1}\left(\boldsymbol{h}_{m+1}, \boldsymbol{t}\right)=\tau_{m}(0, \boldsymbol{t}) \Rightarrow \boldsymbol{G}_{m+1}^{*} \boldsymbol{k}_{m+1}^{*}\left(A_{m+1} e^{i k_{m+1}^{*} h_{m+1}}-B_{m+1} e^{-i k_{m+1}^{*} h_{m+1}}\right)=G_{m}^{*} \boldsymbol{k}_{m}^{*}\left(A_{m}-B_{m}\right)
\end{aligned}
$$

where $\boldsymbol{u}_{\boldsymbol{m}}(\boldsymbol{z}, \boldsymbol{t})$ and $\tau_{\boldsymbol{m}}(\boldsymbol{z}, \boldsymbol{t})$ represent the displacement and shear stress of layer $m$, respectively, and the shear stress is defined as $\tau_{\boldsymbol{m}}=\boldsymbol{G}_{\boldsymbol{m}}^{*} \partial \boldsymbol{u}_{\boldsymbol{m}} / \partial \boldsymbol{z}$, in which the complex shear modulus is expressed as $\boldsymbol{G}_{\boldsymbol{m}}^{*}=\boldsymbol{G}_{\boldsymbol{m}}\left(1+2 \boldsymbol{i} \xi_{\boldsymbol{m}}\right)$. Equations (2)-(3) can be solved to find the wave amplitude factors at layer $m$ as a function of those at layer $m+1$.

$$
\left[\begin{array}{l}
\boldsymbol{A}_{m} \\
\boldsymbol{B}_{m}
\end{array}\right]=\frac{1}{2}\left[\begin{array}{ll}
\left(1+\alpha_{m+1}\right) \boldsymbol{e}^{i k_{m+1}^{*} h_{m+1}} & \left(1-\alpha_{m+1}\right) \boldsymbol{e}^{-i k_{m+1}^{*} h_{m+1}} \\
\left(1-\alpha_{m+1}\right) \boldsymbol{e}^{i k_{m+1}^{*} h_{m+1}} & \left(1+\alpha_{m+1}\right) \boldsymbol{e}^{-i k_{m+1}^{*} h_{m+1}}
\end{array}\right]\left[\begin{array}{l}
\boldsymbol{A}_{m+1} \\
\boldsymbol{B}_{m+1}
\end{array}\right]
$$

in which $\alpha_{\boldsymbol{m}+1}=\frac{\rho_{\boldsymbol{m}+1} \boldsymbol{V}_{\boldsymbol{m}+1}^{*}}{\rho_{\boldsymbol{m}} \boldsymbol{V}_{\boldsymbol{m}}^{*}}$ is the complex impedance ratio between layer $m+1$ and $m, \rho_{\boldsymbol{m}}$ is the mass density of layer $m$, and $\boldsymbol{V}_{\boldsymbol{m}}^{*}=\sqrt{\boldsymbol{G}_{\boldsymbol{m}}^{*} / \rho_{\boldsymbol{m}}}$. By considering a stress-free surface at the top of layer $n$ (i.e., $\tau_{\boldsymbol{n}}(0, \boldsymbol{t})=0$ ), it can be concluded that $\boldsymbol{A}_{n}=\boldsymbol{B}_{n}=\boldsymbol{A}$, where $\boldsymbol{A}$ is a constant. Therefore, the recursive solution shown in equation (4) can be used repeatedly to find the following relationship between the amplitude of the travelling waves in layer $m$ and layer $n$, i.e., the top layer.

$$
\boldsymbol{A}_{m}=\alpha_{m}(\boldsymbol{\theta}, \boldsymbol{f}) \boldsymbol{A}, \boldsymbol{B}_{m}=\beta_{m}(\boldsymbol{\theta}, \boldsymbol{f}) \boldsymbol{A}
$$

where $\alpha_{m}(\boldsymbol{\theta}, \boldsymbol{f})$ and $\beta_{m}(\boldsymbol{\theta}, \boldsymbol{f})$ are scalar valued functions of frequency and the wave model parameter vector, which is denoted by $\boldsymbol{\theta}$. The wave model parameter vector consists of shear wave velocity, density, and damping ratio of the layers. The absolute displacement (or acceleration) FRF (or transfer function) at the top of layer $m$ with respect to the displacement (or acceleration) input at the base is denoted as $\boldsymbol{H}_{\boldsymbol{m}}(\boldsymbol{\theta}, \boldsymbol{f})$ and is derived as 


$$
\boldsymbol{H}_{m}(\boldsymbol{\theta}, \boldsymbol{f})=\frac{\boldsymbol{u}_{m}(0, \boldsymbol{t})}{\boldsymbol{u}_{1}\left(\boldsymbol{h}_{1}, \boldsymbol{t}\right)}=\frac{\alpha_{m}(\boldsymbol{\theta}, \boldsymbol{f})+\beta_{m}(\boldsymbol{\theta}, \boldsymbol{f})}{\alpha_{1}(\boldsymbol{\theta}, \boldsymbol{f}) \boldsymbol{e}^{i \boldsymbol{k}_{1}^{*} h_{1}}+\beta_{1}(\boldsymbol{\theta}, \boldsymbol{f}) \boldsymbol{e}^{-i k_{1}^{*} h_{1}}}
$$

\section{Dispersive model parametrization}

The shear wave propagation in building structures is known to be dispersive, i.e., shear waves of different wavelength propagate at different velocities. The flexural and flexural-shear modes of deformation are most likely the main reason for dispersion of shear waves in a building structure [11-12]. In order to account for the dispersion, an empirical function is proposed in this study to characterize the shear wave phase velocity as a function of frequency at each layer as

$$
V_{m}(f)=\frac{\boldsymbol{a}_{m}^{V}\left(\frac{\boldsymbol{f}}{\boldsymbol{f}_{\max }}\right)}{\boldsymbol{b}_{m}^{V}\left(\frac{\boldsymbol{f}}{\boldsymbol{f}_{\max }}\right)^{2}+\boldsymbol{c}_{m}^{V}\left(\frac{\boldsymbol{f}}{\boldsymbol{f}_{\text {max }}}\right)+1}
$$

in which $f_{\max }$ is the maximum frequency to be considered in the estimation (i.e., $0 \leq \boldsymbol{f} \leq \boldsymbol{f}_{\max }$ ). The phase velocity function has three degrees of freedom; thus, it can be fully characterized by determining the slopes at $f=0$ and $f=f_{\max }$, and the phase velocity at $\boldsymbol{f}=\boldsymbol{f}_{\max }$. To ensure a smooth positive phase velocity function, monotonically increasing with a negative curvature, the following constraints are defined for the phase velocity parameters at each layer.

$$
\boldsymbol{a}_{m}^{V}>0, \boldsymbol{b}_{m}^{V}<1, \boldsymbol{c}_{m}^{V}>0, \boldsymbol{b}_{m}^{V}+\frac{1}{2} \boldsymbol{c}_{m}^{V}>0
$$

Therefore, the phase velocity parameter vector to be estimated for layer $m$ is defined as $\boldsymbol{\theta}_{m}^{V}=\left[\boldsymbol{a}_{m}^{V}, \boldsymbol{b}_{m}^{V}, \boldsymbol{c}_{m}^{V}\right]$. Consequently, the phase velocity parameter vector for the multilayer model shown in Figure 1 is defined as $\boldsymbol{\theta}^{V}=\left[\boldsymbol{\theta}_{1}^{V}, \boldsymbol{\theta}_{2}^{V}, \ldots, \boldsymbol{\theta}_{n}^{V}\right]$.

\section{Sequential Bayesian estimation of wave model parameters using FRFs}

The frequency domain is discretized into $N$ sampling points with frequency spacing $\Delta f=f_{\max } / N-1$. At each sampling point, the FRFs are estimated from the analytical model and measurement data. The predicted FRF from the analytical model at the top of layer $m$ at the $k^{\text {th }}$ frequency sampling point is denoted as $\boldsymbol{H}_{m k}(\boldsymbol{\theta})=\boldsymbol{H}_{m}(\boldsymbol{\theta},(\boldsymbol{k}-1) \times \Delta \boldsymbol{f})=\boldsymbol{H}_{m \boldsymbol{k}}^{\boldsymbol{R}}+\boldsymbol{i} \boldsymbol{H}_{m \boldsymbol{k}}^{\boldsymbol{I}}$, where $\boldsymbol{H}_{m k}^{\boldsymbol{R}}$ and $\boldsymbol{H}_{\boldsymbol{m} \boldsymbol{k}}^{\boldsymbol{I}}$ are the real and imaginary parts of $\boldsymbol{H}_{\boldsymbol{m} \boldsymbol{k}}$, respectively, and $i$ denotes the imaginary unit. The values of the estimated FRFs can be stacked in a tall vector, as

$$
\hat{\mathbf{y}}_{k}(\boldsymbol{\theta})=\left[\left[\left[\begin{array}{ll}
H_{11}^{R} & H_{11}^{I}
\end{array}\right]\left[\begin{array}{ll}
H_{12}^{R} & H_{12}^{I}
\end{array}\right] \quad \cdots \quad\left[\begin{array}{ll}
H_{1 k}^{R} & H_{1 k}^{I}
\end{array}\right]\right] \cdots\left[\left[\begin{array}{ll}
H_{n 1}^{R} & H_{n 1}^{I}
\end{array}\right]\left[\begin{array}{ll}
H_{n 2}^{R} & H_{n 2}^{I}
\end{array}\right] \cdots\left[\begin{array}{ll}
H_{n k}^{R} & H_{n k}^{I}
\end{array}\right]\right]\right]^{T}
$$

in which $\hat{\mathbf{y}}_{\boldsymbol{k}} \in \mathbb{R}^{(2 \boldsymbol{k} n) \times 1}(1 \leq \boldsymbol{k} \leq \boldsymbol{N})$ is the predicted FRF vector, and $n$ depicts the number of layers. Similarly, the measured FRFs are estimated at the same frequency sampling points and stacked in the measured FRF vector, $\mathbf{y}_{k}$, as

$$
\left.\left.\mathbf{y}_{k}(\boldsymbol{\theta})=\left[\left[\begin{array}{ll}
{\left[\hat{\hat{G}}_{11}^{R}\right.} & \hat{\hat{G}}_{11}^{I}
\end{array}\right]\left[\begin{array}{ll}
\hat{\hat{G}}_{12}^{R} & \hat{\hat{G}}_{12}^{I}
\end{array}\right] \quad \cdots\left[\begin{array}{cc}
\hat{\hat{G}}_{1 k}^{R} & \hat{\hat{G}}_{1 k}^{I}
\end{array}\right]\right] \ldots\left[\begin{array}{ll}
\hat{\hat{G}}_{n 1}^{R} & \hat{\hat{G}}_{n 1}^{I}
\end{array}\right]\left[\begin{array}{ll}
\hat{\hat{G}}_{n 2}^{R} & \hat{\hat{G}}_{n 2}^{I}
\end{array}\right] \ldots\left[\begin{array}{ll}
\hat{\hat{G}}_{n k}^{R} & \hat{\hat{G}}_{n k}^{I}
\end{array}\right]\right]\right]^{T}
$$

in which $\hat{\hat{G}}_{m k}$ is the estimated transfer functions (or FRF) between the output measurements at the top of layer $m$ and the input excitations at the $k^{\text {th }}$ frequency sampling point. The FRFs are estimated as

$$
\hat{\hat{G}}_{m}(f)=\frac{\hat{\hat{\Phi}}_{a_{m} a_{g}}(f)}{\hat{\hat{\Phi}}_{a_{g} a_{g}}(f)}
$$


where $\hat{\hat{\Phi}}_{a_{m} a_{g}}(f)=$ cross spectral density between the floor absolute acceleration response measured at level $m$ of a building structure, $\boldsymbol{a}_{m}(t)$, and the ground acceleration input, $\boldsymbol{a}_{g}(t)$, and $\hat{\hat{\Phi}}_{a_{g} a_{g}}(f)=$ auto spectral density estimate of $\boldsymbol{a}_{g}(t)$. The auto/cross spectral densities are estimated using the Welch's method [13].

The unknown wave model parameter vector, $\boldsymbol{\theta}$, is modeled as a random vector (denoted by $\boldsymbol{\Theta}$ ), whose values are uncertain and their uncertainties are characterized by a probability distribution function (pdf). The prior knowledge about the wave model parameter vector is modeled as a prior pdf. The prior pdf of $\boldsymbol{\Theta}$ can be updated to posterior pdf by extracting information from the discrepancies between the predicted and measured FRFs through the Bayes' rule. The discrepancy between the predicted and measured FRFs is modeled as

$$
\mathbf{y}_{k}=\hat{\mathbf{y}}_{k}(\boldsymbol{\theta})+\mathbf{v}_{k}(\boldsymbol{\theta})
$$

in which $\mathbf{v}_{\boldsymbol{k}}=$ simulation error vector, which is modeled herein as an independent and identically distributed Gaussian white noise. Assuming that the prior pdf of the wave model parameter vector is Gaussian, the posterior pdf can be derived as [14]

$$
\boldsymbol{p}\left(\boldsymbol{\theta} \mid \mathbf{y}_{k}\right)=\frac{1}{\boldsymbol{p}\left(\mathbf{y}_{k}\right)} \times \frac{\boldsymbol{e}^{-\frac{1}{2}\left(\mathbf{v}_{k}(\boldsymbol{\theta})\right)^{T} \mathbf{R}_{k}^{-1}\left(\mathbf{v}_{k}(\boldsymbol{\theta})\right)}}{(2 \pi)^{k n}\left|\mathbf{R}_{k}\right|^{1 / 2}} \times \frac{\boldsymbol{e}^{-\frac{1}{2}\left(\boldsymbol{\theta}-\hat{\boldsymbol{\theta}}^{-}\right)^{T}\left(\hat{\mathbf{P}}^{-}\right)^{-1}\left(\boldsymbol{\theta}-\hat{\boldsymbol{\theta}}^{-}\right)}}{(2 \pi)^{n_{\boldsymbol{\theta}} / 2}\left|\hat{\mathbf{P}}^{-}\right|^{1 / 2}}
$$

where $\mathbf{R}_{\boldsymbol{k}} \in \mathbb{R}^{(2 \boldsymbol{k} \boldsymbol{n}) \times(2 \boldsymbol{k} \boldsymbol{n})}=$ covariance matrix of the simulation error vector $\mathbf{v}_{\boldsymbol{k}} ; \hat{\boldsymbol{\theta}}^{-}$is the prior mean vector, and $\hat{\mathbf{P}}^{-} \in \mathbb{R}^{n_{\theta} \times n_{\theta}}$ is the prior covariance matrix of the wave model parameter vector. Since $\mathbf{v}_{\boldsymbol{k}}$ is modeled as an independent and identically distributed Gaussian white noise, $\mathbf{R}_{\boldsymbol{k}}$ will be a diagonal matrix.

Equation (13) results in a non-Gaussian distribution for the posterior pdf of wave model parameter vector. To simplify the mathematical derivations, the posterior pdf can be gaussianized using a first-order approximation. The first order Taylor series expansion of $\mathbf{v}_{\boldsymbol{k}}(\boldsymbol{\theta})$ with respect to $\boldsymbol{\theta}$ at $\hat{\boldsymbol{\theta}}^{-}$can be expressed as

$$
\mathbf{v}_{k}(\boldsymbol{\theta})=\mathbf{y}_{k}-\hat{\mathbf{y}}_{k}(\boldsymbol{\theta}) \cong \mathbf{y}_{k}-\hat{\mathbf{y}}_{k}\left(\hat{\boldsymbol{\theta}}^{-}\right)-\mathbf{C}\left(\boldsymbol{\theta}-\hat{\boldsymbol{\theta}}^{-}\right)
$$

in which $\mathbf{C}=\left.\frac{\partial \hat{\mathbf{y}}_{k}(\boldsymbol{\theta})}{\partial \boldsymbol{\theta}}\right|_{\boldsymbol{\theta}=\hat{\boldsymbol{\theta}}^{-}}$is the model response sensitivity matrix and can be computed by analytically differentiating equation (6) with respect to $\boldsymbol{\theta}$. Substitution of equation (14) into equation (13) results in the following equations to update the mean vector and covariance matrix of the gaussanized posterior pdf of $\boldsymbol{\Theta}$.

$$
\hat{\boldsymbol{\theta}}^{+}=\hat{\boldsymbol{\theta}}^{-}+\mathbf{K}\left(\mathbf{y}_{k}-\hat{\mathbf{y}}_{k}\left(\hat{\boldsymbol{\theta}}^{-}\right)\right), \hat{\mathbf{P}}^{+}=\left(\mathbf{C}^{T} \mathbf{R}_{k}^{-1} \mathbf{C}+\left(\hat{\mathbf{P}}^{-}\right)^{-1}\right)
$$

in which $\mathbf{K}$ is the Kalman gain matrix and can be expressed as [15]

$$
\mathbf{K}=\left(\mathbf{C}^{T} \mathbf{R}_{k}^{-1} \mathbf{C}+\left(\hat{\mathbf{P}}^{-}\right)^{-1}\right)^{-1} \mathbf{C}^{T} \mathbf{R}_{k}^{-1}
$$

The posterior estimates obtained from equation (15) should be corrected to satisfy the constraints shown in equation (8). For this purpose, the posterior Gaussian pdf that is resulted from equation (15) is truncated at the constraint edges. The constrained posterior mean vector and covariance matrix are then calculated using the truncated pdf. To this end, the wave model parameter space is transformed into a new space to decouple the constraints, so that each constraint equation will involve only one parameter in the transformed space. Then, the pdf of the transformed single parameter is truncated to correct for constraints and the corrected mean and variance (obtained from the truncated pdf) are transferred back to the wave model parameter space. More details about constrained recursive estimation can be found in [15] and [16]. 


\section{NUMERICAL CASE STUDY}

The benchmark building used for this case study is a 52-story building structure, instrumented as a part of the community seismic network (CSN) project [17-18]. A detailed three-dimensional linear-elastic model of the structural system has been developed in the structural analysis software ETABS [19] based on the available structural drawings [20]. The model is used to generate simulated structural response data for the purpose of validating the wave model parameter estimation method presented in this study. As mentioned before, each layer in the multilayer Kelvin-Voigt shear beam model represents a single story or group of stories of the building structures. In this case study, two different layering configurations are considered as presented in Table 1. In the first layering configuration, which is referred to as Case \#1 model, the building structure is modeled as a 51-layer shear beam model. The basement and penthouse stories are combined together and modeled as a single layer. Every two layers between layers \#2 and \#49 have the same phase velocity and damping ratio parametrization. Therefore, 27 sets of phase velocity and damping ratio parameters, or $27 \times(3+2)=135$ unknown parameters, characterize Case \#1 model. In contrast, Case \#2 model consists of a 51-layer shear beam model, in which each layer has an independent parametrization to characterize the phase velocity and damping ratio. Therefore, Case \#2 model has $51 \times(3+2)=255$ unknown phase velocity and damping ratio parameters. Both Case \#1 and Case \#2 models have the same parametrization for mass density, as listed in the last column of Table 1. Since the mass distribution along the height of the building structure is piecewise uniform, many layers are expected to have similar mass densities. Furthermore, following equations (5) and (6), it can be concluded that the analytical FRFs depend on the relative mass density of the layers. To simplify the estimation process, the mass density of the top layer (i.e., layer \#51) is set as a unit constant (i.e., $\rho_{7}=1$ ). Thus, six unknown mass density parameters (i.e., $\rho_{1}$ to $\rho_{6}$ ) are used to characterize the mass distribution for both Case \#1 and Case \#2 models. In fact, each mass density parameter represents the relative mass density of the corresponding layer with respect to layer \#51. The last three columns in Table 1 list the labels for parameter sets that characterize phase velocity (and damping ratio) and mass density.

Table 1. ETABS model parameters and layering configurations used for multilayer Kelvin-Voigt dispersive shear beam model identification.

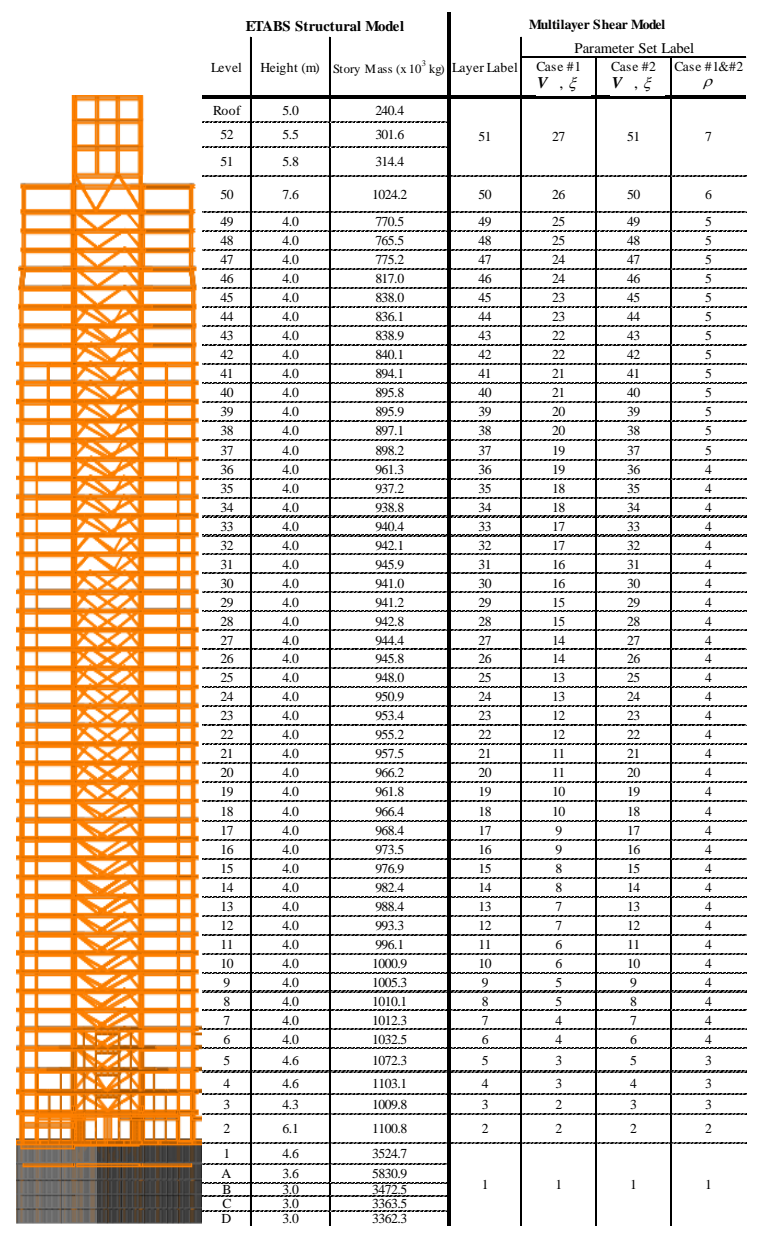




\section{Structural system identification}

A 240-seconds long, 5\% g root-mean-square (RMS) Gaussian white noise acceleration time history sampled at $50 \mathrm{~Hz}$ is used as the base acceleration time history to simulate the floor absolute acceleration responses of the building structure. Although such a long broadband base excitation is unrealistic in the real-world, it has been considered herein to provide an ideal condition for a proof-of-concept study to validate the performance of the proposed wave model parameter estimation method. The time histories of the floor absolute acceleration response in the direction of base motion are computed at the geometric center of the floors. No artificial measurement noise is added to the simulated responses. Spectral analysis method based on the Welch's averaging method [21], is used to find the FRF of the floor absolute acceleration responses with respect to the input base acceleration. The acceleration time histories are divided into five estimation windows with 50\% overlap. Each window is weighted by a Hann function. The auto/cross spectra densities are estimated across each window and averaged over the five estimation windows. The FRFs are estimated in the frequency range of 0 to $6 \mathrm{~Hz}$, with a frequency spacing of $\Delta f \cong 0.0244 \mathrm{~Hz}$.

The sequential Bayesian estimation algorithm described earlier is used to estimate the wave model parameters. The initial values for the phase velocity and damping ratio parameters are the same for all layers in both Case \#1 and Case \#2 models. The initial value for the phase velocity parameter vector is selected as $\boldsymbol{\theta}_{m \text {,initial }}^{V}=[12000,-7,46]$ for each layer. The initial values for the relative mass densities can be selected based on the prior information about the mass distribution along the building height. Here, they are initialized based on the story masses defined in the model as $\boldsymbol{\theta}_{\text {initial }}^{\rho}=\left[\rho_{1}, \rho_{2}, \ldots, \rho_{6}, \rho_{7}\right]=[16.0,3.4,4.5,4.6,4.0,2.5,1.0]$, where $\rho_{7}=1.0$ is constant. Other different initial values for the wave model parameters have also been investigated to check the consistency of the estimation results. The prior wave model parameter covariance matrix is defined as a diagonal matrix, where the diagonal entries are selected by assuming a uniform $20 \%$ prior coefficient of variation (COV) for each wave model parameter..

The wave model parameters are estimated considering both dispersive and nondispersive models. For the nondispersive system identification, the dispersive relation in equation (7) is simplified as $\boldsymbol{V}_{\boldsymbol{m}}(\boldsymbol{f})=\boldsymbol{a}_{\boldsymbol{m}}^{V}$ where $\boldsymbol{a}_{\boldsymbol{m}}^{V}$ denotes the constant (nondispersive) phase velocity for layer $m$. The same estimation procedure is used for the nondispersive identification. The initial values for the phase velocity and damping ratio for the nondispersive model are selected as $\boldsymbol{\theta}_{\boldsymbol{m} \text {,initial }}^{V}=275.9 \mathrm{~m} / \mathrm{s}$ and $\boldsymbol{\theta}_{m, \text { initial }}^{\xi}=2.1 \%$, respectively, which are equivalent to the initial phase velocity and damping ratio of the dispersive model at $f=3 \mathrm{~Hz}$.

Figure 2 compares the measured, initial, and estimated FRFs for Case \#1 model at two layers: layers \#1, and \#33. The real and imaginary parts of the FRFs are plotted separately. As can be observed, the initial FRFs are significantly different from the measured FRFs, while the estimated FRFs using the dispersive model match the measurements with good accuracy. The identified dispersive model captures correctly all the mode shapes in the frequency range considered for estimation. This verifies the correct performance of the estimation algorithm in steering the initial model to reduce its prediction discrepancies with the measurements. The estimated FRFs are plotted for both the dispersive and nondispersive models. It can be concluded that the FRFs of the identified dispersive model match the measurements better than the nondispersive model and the identified dispersive model provides a better prediction of the measured FRFs.

Figure 3 compares the estimation results for Case \#1 and Case \#2 dispersive models. This figure compares the final estimates of the phase velocity (Figure 3(a)) and damping ratio (Figure 3(b)) - both evaluated at $f=3 \mathrm{~Hz}$ - the relative mass density profile (Figure 3(c)), and the relative root mean square errors (RRMSE) of the estimated FRFs (Figure 3(d)). The phase velocity and relative mass density estimates for the two models are comparable along the height of the building. Nevertheless, the damping ratio estimates show non-negligible differences. The inconsistency in the identified damping ratios is most likely due to the weak identifiability of the damping parameters. The RRMSE for the estimated FRF at level $m$ is defined as

$$
\boldsymbol{R R M S E}_{m}(\%)=\sqrt{\frac{\sum_{k=1}^{N}\left|\boldsymbol{H}_{m k}(\hat{\boldsymbol{\theta}})-\hat{\hat{\boldsymbol{G}}}_{m k}\right|^{2}}{\sum_{k=1}^{N}\left|\hat{\boldsymbol{G}}_{m k}\right|^{2}}} \times 100
$$

Figure 3(a) shows an unphysical fluctuation pattern in the phase velocity estimates along the building height, especially at lower and upper levels. The reason for this pattern can be justified by considering the estimation bias and the effects of model 
uncertainty. Obviously, the response behavior of the structural model is physically different from a multilayer dispersive Kelvin-Voigt model, no matter how the wave model parameters are tuned. The difference is more dominant where there are sudden changes (or discontinuities) in the lateral stiffness along the height of the building. The 52-story building model has stiffness contrasts at the lower levels, where the upper ground braced frame structure connects to the basement shear wall structure, and at the penthouse location. These contrasts result in a mismatch between the response behavior of the structural model and the dispersive shear beam model, which in turn, results in biased and unphysical estimation of phase velocities. Figure 3(a) shows that the fluctuation pattern is more significant in the lower and upper levels of the building than the middle levels (i.e., layer \#30 to \#40), where the response behavior of the structural system is close to a shear beam model. It should be noted that by including higher modes in the estimation (i.e., considering larger frequency estimation range) the estimation bias will most likely be reduced, but will never be eliminated completely due to the effects of model uncertainties.
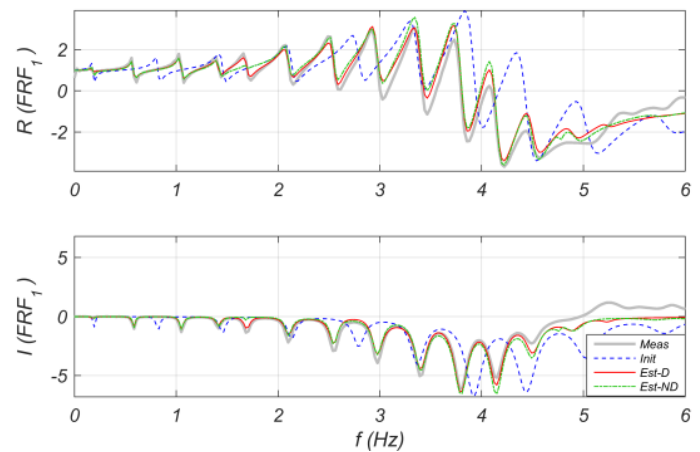

(a)
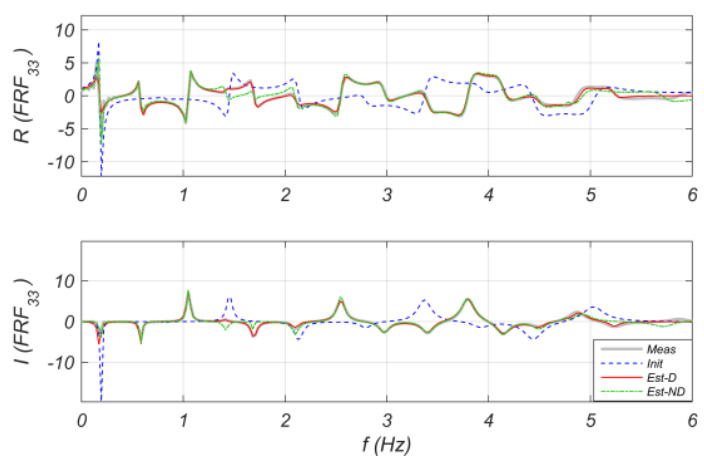

(b)

Figure 2: Comparison of the measured (Meas), initial (Init), and estimated (Est) real (R) and imaginary (I) parts of the FRF for Case \#1 model at two layers: (a) layer \#1, (b) layer \#33. The estimated FRFs are plotted for both the dispersive

(Est-D) and nondispersive (Est-ND) models.

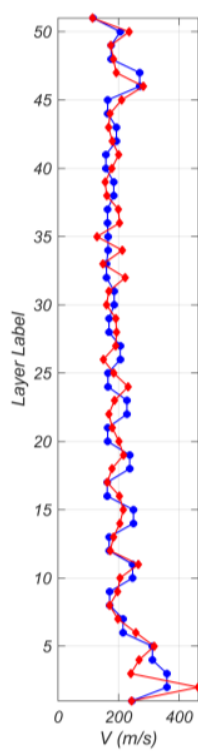

(a)

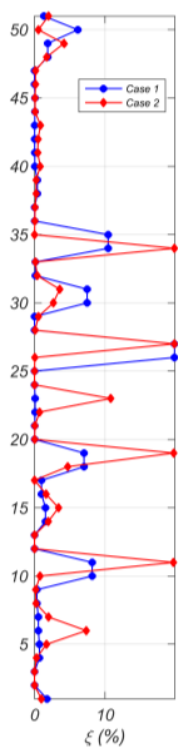

(b)

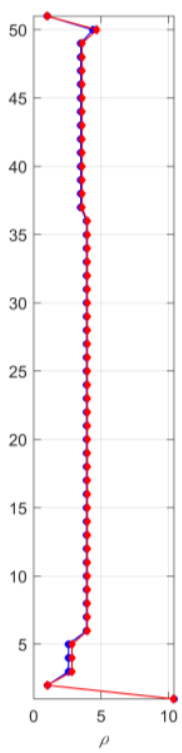

(c)

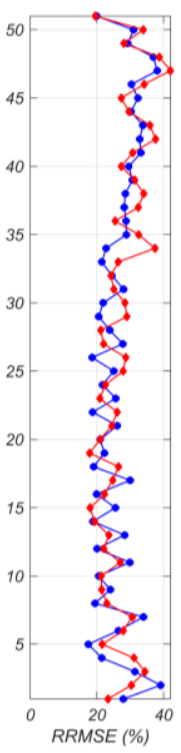

(d)

\begin{abstract}
Figure 3: Comparison of estimation profiles for Case \#1 and Case \#2 models; (a) final estimates of phase velocity at $\boldsymbol{f}=3 \mathrm{~Hz}$; (b) final estimates of damping ratio at $\boldsymbol{f}=3 \mathrm{~Hz}$; (c) final estimates of relative mass density; $(d)$ relative root mean square error (RRMSE) of the estimated FRFs.
\end{abstract}

\section{CONCLUSIONS}

This study proposed a sequential Bayesian estimation algorithm for estimating the parameters characterizing the propagation of seismic waves through a continuous, spatially non-homogenous viscoelastic solid. These parameters include those that characterize the phase velocity and damping ratio as a function of frequency, and the relative mass densities of a multilayer Kelvin-Voigt model. While the proposed Bayesian estimation method is general and can be applied to other waveform inversion problem, it was validated using a building system identification problem in this study. The case study showed the correct performance and accuracy of the proposed structural system identification approach, which can potentially be used for detection and localization of structural damage at the story level of building structures. 


\section{ACKNOWLEDGMENTS}

Support of this research provided by the Terrestrial Hazard Observation and Reporting (THOR) Center at Caltech is gratefully acknowledged.

\section{REFERENCES}

[1] E. Şafak, "Wave-Propagation Formulation of Seismic Response of Multistory Buildings," ASCE Journal of Structural Engineering, vol. 125, no. 4, pp. 426-437, 1999.

[2] M. I. Todorovska, S. S. Ivanović, and M. D. Trifunac, "Wave Propagation in a Seven-Story Reinforced Concrete Building: I. Theoretical Models," Soil Dynamics and Earthquake Engineering, vol. 21, no. 3, pp. 211-223, 2001.

[3] H. Kawakami and M. Oyunchimeg, "Normalized Input-Output Minimization Analysis of Wave Propagation in Buildings," Engineering Structures, vol. 25, no. 11, pp. 1429-1442, 2003.

[4] R. Snieder and E. Şafak, "Extracting the Building Response Using Seismic Interferometry: Theory and Application to the Millikan Library in Pasadena, California," Bulletin of the Seismological Society of America, vol. 96, no. 2, pp. 586$598,2006$.

[5] M. D. Kohler, T. H. Heaton, and S. C. Bradford, "Propagating Waves in the Steel, Moment-Frame Factor Building Recorded during Earthquakes," Bulletin of the Seismological Society of America, vol. 97, no. 4, pp. 1334-1345, 2007.

[6] M. I. Todorovska and M. T. Rahmani, "System Identification of Buildings by Wave Travel Time Analysis and Layered Shear Beam Models - Spatial Resolution and Accuracy," Structural Control and Health Monitoring, vol. 20, no. 5, pp. 686-702, 2013.

[7] M. I. Todorovska and M. D. Trifunac, "Earthquake Damage Detection in the Imperial County Services Building III: Analysis of Wave Travel Times via Impulse Response Functions," Soil Dynamics and Earthquake Engineering, vol. 28, no. 5, pp. 387-404, 2008.

[8] M. I. Todorovska and M. D. Trifunac, "Impulse Response Analysis of the Van Nuys 7-Storey Hotel During 11 Earthquakes and Earthquake Damage Detection," Structural Control and Health Monitoring, vol. 15, no. 1, pp. 90-116, 2007.

[9] M. Rahmani and M. I. Todorovska, "Structural Health Monitoring of a 54-Story Steel-Frame Building using a Wave Method and Earthquake Records," Earthquake Spectra, vol. 31, no. 1, pp. 501-525, 2015.

[10] S. L. Kramer, Geotechnical Earthquake Engineering.: Pearson Education, 1996.

[11] M. Ebrahimian, M. Rahmani, and M. I. Todorovska, "Nonparametric Estimation of Wave Dispersion in High-Rise Buildings by Seismic Interferometry," Earthquake Engineering and Structural Dynamics, vol. 43, no. 15, pp. 2361$2375,2014$.

[12] M. Ebrahimian and M. I. Todorovska, "Wave Propagation in a Timoshenko Beam Building Model," ASCE Journal of Engineering Mechanics, vol. 140, no. 5, p. 04014018, 2014.

[13] S. M. Alessio, Digital Signal Processing and Spectral Analysis for Scientists: Concepts and Applications. Switzerland: Springer International Publishing, 2016.

[14] H. Ebrahimian, R. Astroza, J. P. Conte, and R. A. de Callafon, "Nonlinear Finite Element Model Updating for Damage Identification of Civil Structures using Batch Bayesian Estimation," Mechanical Systems and Signal Processing, vol. 10.1016/j.ymssp.2016.02.002, 2016.

[15] D. Simon, Optimal State Estimation: Kalman, H-Infinity, and Nonlinear Approaches. Hoboken, NJ: John Wiley \& Sons, 2006.

[16] D. Simon and D. L. Dimon, "Constrained Kalman Filtering via Density Function Truncation for Turbofan Engine Health Estimation," International Journal of Systems Science, vol. 41, no. 2, pp. 159-171 , 2010.

[17] R. W. Clayton et al., "Community Seismic Network: A Dense Array to Sense Earthquake Strong Motions," Seismological Research Letters, vol. 86, no. 5, pp. 1354-1363, 2015.

[18] R. Clayton, T. Heaton, M. Chandy, A. Krause, M. Kohler, J. Bunn, R. Guy, M. Olson, M. Faulkner, M.-H. Cheng, L. Strand, R. Chandy, D. Obenshain, A. Liu and M. Aivazis, "Community Seismic Network," Annals of Geophysics, vol. 54, no. 6, pp. 37-747, 2011.

[19] Computers and Structures Inc., "Extended Three Dimensional Analysis of Building Systems, ETABS Ver. 15.2 ," Berkeley, CA, 2015.

[20] M. Kohler, A. Massari, T. H. Heaton, H. Kanamori, E. Hauksson, R. Guy, R. W. Clayton, J. Bunn and K. M. Chandy, "Downtown Los Angeles 52-Story High-Rise and Free-Field Response to an Oil Refinery Explosion," Earthquake Spectra, pp. DOI: 10.1193/062315EQS101M, 2016.

[21] A. K. Tangirala, Principles of System Identification: Theory and Practice. Boca Raton, FL: CRC Press, 2015. 